\title{
A CELL-SUSPENSION MIXER
}

\author{
BY \\ G. A. MATTHEWS \\ From the West Herts Hospital, Hemel Hempstead
}

(RECEIVED FOR PUBLICATION APRIL 11, 1951)

For the fundamental idea of mixing cell suspensions by rotation at a low speed I am indebted to Dr. J. V. Dacie's book Practical Haematology (1950).

The machine depicted here carries a variety of spring clips, to take different sized containers, on a turntable inclined at an angle of $45^{\circ}$. The head rotates slowly so that the air bubbles in the containers travel completely up and down with each revolution. The mixer is electrically driven and is silent in operation. There is an on-off switch, but the rotation can be stopped briefly by hand without using this if desired.

The following advantages may be claimed for the use of this machine in haematology. Red and white cell suspensions can be made with $20 \mathrm{ml}$. and $50 \mathrm{ml}$. pipettes, thus dispensing with the less accurate bulb pipettes and reducing the error due to dilution. The tubes are then clipped to the mixer and after a lapse of three minutes can be removed as and when required for charging counting chambers with Pasteur pi pet tes. If Wintrobe's method of doing blood counts is used the mixing of the initial oxalated blood sample is done by the machine as well. The setting up of a number of sedimentation tubes is greatly facilitated.

The machine can be used to defibrinate blood given a container with a snare attached to the cap to collect the fibrin.

It has been found that very even mixing of cell-suspensions is achieved, with a saving of time proportionate to the number of blood counts undertaken.

The machine can be obtained from Messrs. Hawkesley \& Sons, Ltd., 17, New Cavendish Street, London, W.1.

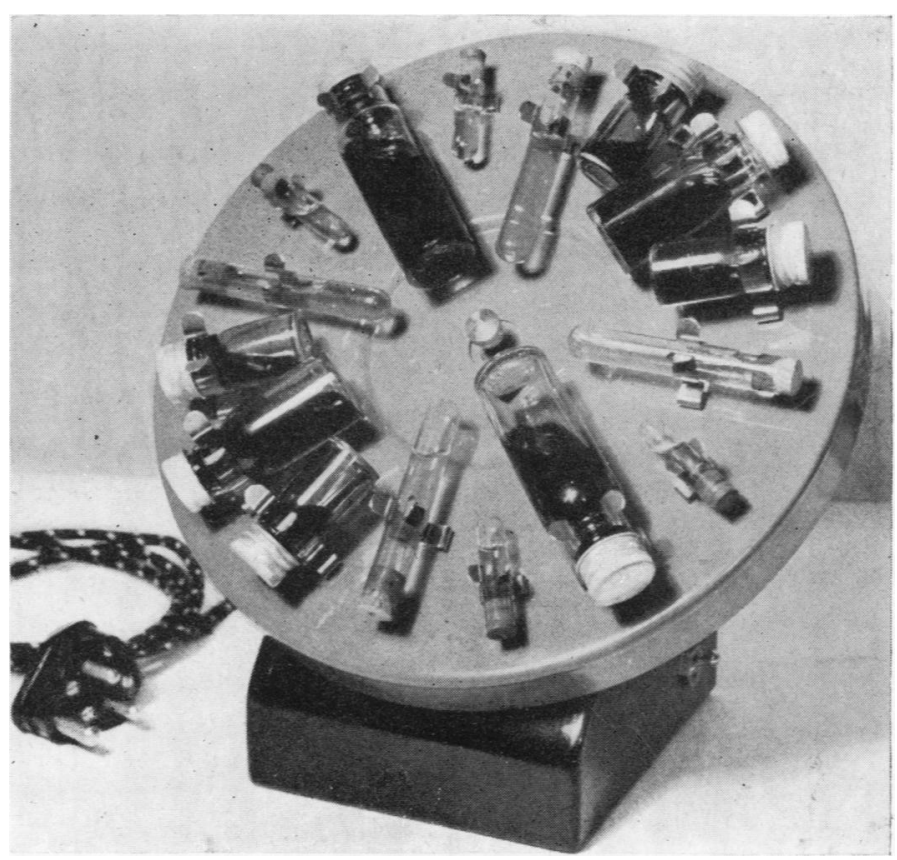


The Morbid Anatomy of Tuberculosis in Old Age. Roulet, F. (1950). Ann. Méd., 51, 69.

In 270 necropsies carried out at the University of Basle on subjects over 60 years old, frank evidence of tuberculosis was present, although it was the cause of death in only 90 cases. The active focus was found in 245 cases around an old calcified or ossiried nodule in the tracheo-bronchial lymph nodes; The lungs in these cases revealed no active primary focus, though an old, calcified, inactive lesion was often discovered. It is argued that active tuberculosis in old age is frequently caused by reactivation of an old lesion in the lymph nodes around the bifurcation of the trachea, possibly due to the chronic inflammation of the lower tracher and main bronchi, which is extremely common in old people. Tuberculosis of the tracheo-bronchial nodes frequently leads to complications: of the 245 cases of tracheobronchial-node infection, there was also frank tuberculous infection of the lungs in 35: in 12 cases there was a generalized haematogenous spread either from a focus in the tracheo-bronchial nodes or from an alternative site of active infection; in 13 cases there were foci both in the tracheobronchial nodes and elsewhere, but no generalized spread; and in 25 cases haematogenous spread had occurred with tracheobronchial nodes as the only possible primary source. In the remaining 160 cases the infection was confined to the tracheobronchial nodes.

Speaking generally, miliary tuberculosis develops more rapidly in the old than at other ages. The appearances are classical, though the tubercles may be rather largeas big as a pea or a hazel nut. In the whole series there were 16 cases of miliary spread amongst the 43 cases of frank pulmonary tuberculosis. Tuberculosis of the serous cavities is not uncommon in the elderly. Pulmonary tuberculosis may be of the chronic apical fibro-caseous type with cavities, or of the ulcerative caseous type of acute tuberculosis, the frequent occur- rence of which in old people may possibly be due to reduced general resistance and antibody response.

\section{Glomerulonephrosis. A Morphologic Manifestation of Renal Cortical Ische- mia in Toxic Oliguria and Lower Nephron Nephrosis. French, A. J. (1950). Arch. Path., 49, 43.}

The histopathological findings in the kidneys of 20 specially selected cases of glomerulonephrosis are described, special stress being laid on the glomerular changes. This study was undertaken in the hope of explaining the anuria or oliguria which accompanies lower nephron nephrosis (" tubular" or " toxic" nephritis), in which condition, on physiological grounds, there should be polyuria if a tubular lesion alone be present. For this purpose it was essential to eliminate cases of primary or secondary renal disease, eclampsia, and cardiovascular hypertension.

The changes found in the glomeruli, tubules, and medulla in sections of the kidneys (mostly stained with haematoxylin and eosin) are tabulated. Glomerular ischaemia, together with thickening of the capillary walls, was found in every case. The presence of granular material staining greyblue in the glomerular spaces and proximal tubules was noted, this being regarded as the precursor of the protein casts seen in the distal convoluted tubules in all except one of these cases. $\quad R . B . T$. Baldwin.

Corrections.-Dr. Varadi writes: I shall be obliged if you will print the following corrections to my article "Combined Fragility Test for Congenital Haemolytic Jaundice" (J. clin. Path., 4,221 ). On page 222 in paragraph 3 read $0.021 \%$ instead of $0.21 \% \mathrm{NaCl}$, and, on page 224 paragraph 2 of the Appendix, marrow puncture instead of culture.

We much regret that in the description of a cell-suspension mixer by Dr. Matthews (J. clin. Path. 4,383 ) the pipettes were described as $20 \mathrm{ml}$. and $50 \mathrm{ml}$. These should be $20 \mathrm{c} . \mathrm{mm}$. and 50 c.mm. 